\title{
LA INTEGRACIÓN ACADÉMICA DE LOS ESTUDIANTES UNIVERSITARIOS COMO FACTOR DETERMINANTE DEL ABANDONO DE CORTO PLAZO. UN ANÁLISIS EN EL SISTEMA DE EDUCACIÓN SUPERIOR A DISTANCIA DEL ECUADOR
}

\author{
(ACADEMIC INTEGRATION OF UNIVERSITY STUDENTS AS A DETERMINING FACTOR OF \\ STUDENT DROPOUT IN THE SHORT TERM. AN ANALYSIS OF THE DISTANCE EDUCATION \\ SYSTEM IN ECUADOR)
}

Luis Fabian Moncada Mora

Universidad Técnica Particular de Loja, Ecuador

\section{RESUMEN}

El presente estudio aborda el problema del abandono de los estudios universitarios desde el punto de vista del nivel de integración que logran los estudiantes. Este enfoque se utiliza con el fin de encontrar el factor con la mayor cantidad de información para explicar la decisión de permanecer o abandonar los estudios. La caracterización teórica del fenómeno se convierte en la principal novedad para la teoría del abandono y, en función de ello se determina el alto grado de incidencia que tienen los resultados inmediatos, como medida de integración académica, en la decisión de permanecer o abandonar. Posteriormente, la información estadística se agrupa de tal forma que siguiendo los lineamientos y expectativas, expuestas en la teoría, se puedan encontrar los comportamientos característicos del fenómeno. Para la generación de la evidencia empírica se trabajó con la información de 6.715 estudiantes, pertenecientes a la cohorte abril - agosto 2012 de la Universidad Técnica Particular de Loja que representan el $82 \%$ de la población estudiantil del Ecuador matriculada en el sistema de estudios a distancia. La evidencia se trabajó en dos aspectos principales: primero, la descripción de los datos y aplicación de la caracterización; en esta fase se determina que el 40,6\% de los estudiantes abandonan por motivos académicos, el 8,9\% abandonan por motivos no académicos, el 32,6\% permanecen por motivos académicos y el $17,5 \%$ permanecen por motivos no académicos; y segundo, se estiman modelos probabilísticos que confirman la relación negativa que existe entre los resultados inmediatos, como media de la integración académica, y la decisión de abandonar.

Palabras claves: educación a Distancia, tasa de abandono, estudiantes de pregrado, sistema educativo. 


\begin{abstract}
This study addresses the problem of the abandonment (drop out) of university studies from the point of view of the level of student integration. This approach is used with the aim of locating the factor(s) that provide(s) the greatest amount of information regarding the decision to continue or to abandon one's studies. The theoretical characterization of this phenomenon has become one of the underlying factors that are used to explain the theory behind students dropping out of their courses. Based on this theory, we can determine the level of incidence of student drop outs- and determine the (high) incidence - where immediate results serve as a means of measuring academic integration and the decision to continue or to quit one's studies. Statistical information is grouped in such a way that it is possible to follow the guidelines and expectations outlined in the theory-and also helps us to understand typical characteristics of this phenomenon. To generate empirical evidence information, we worked with data from 6,715 students, who belonged to the April-August 2012 cohort at the Universidad Técnica Particular de Loja, and which represents $82 \%$ of the student population of Ecuador who are enrolled in the distance learning system. Evidence for this study was obtained from the following sources: first, there was a description of the data and an application of the characterization process. In this phase, it was determined that $40.6 \%$ of students dropped out for academic reasons; 8.9\% dropped out for non-academic reasons; $32.6 \%$ stayed for academic reasons; and $17.5 \%$ remained for non-academic reasons. Second, we estimated probabilistic models that confirmed the negative relationship between the immediate results (calculated as an average of academic integration), and factors concerning the decision to quit one's studies.
\end{abstract}

Keywords: Distance Education - Dropout Rate - Undergraduate Students - Educational system

El abandono de los nuevos estudiantes universitarios, independientemente de la modalidad, es un fenómeno que en mayor o menor medida afecta a todos los países. Al final del primer periodo es cuando se tiene la mayor tasa de abandono (Corominas Rovira, 2001); este hecho, sin duda involucra directamente a todos los agentes del sistema educativo (Tinto, 2004). En este proceso el principal afectado es el mismo estudiante que no encuentra la motivación necesaria para permanecer y decide privilegiar otros aspectos de su vida, dejando en segundo plano su formación profesional.

Las causas o argumentos que tienen los estudiantes para tomar la decisión de abandonar son tan diversas como difíciles de cuantificar. La mayoría de los estudios del abandono estudiantil universitario se dedican a especular y llegar a causas tan particulares que al final pueden afectar a una minoría de la población estudiantil, si seguimos esta línea de análisis en educación a distancia, por el propio perfil del estudiante, las causas se diversificarán e incrementarán, volviendo más difícil la labor de buscar soluciones que ayuden a minimizar los efectos de este fenómeno. 
En el Ecuador la demanda de educación superior en el sistema de estudios a distancia ha tenido un crecimiento secular con una tasa promedio del 10\% semestral; este importante crecimiento se ve fuertemente afectado por la desalentadora tasa de abandono que en promedio afecta al 50\% de cada cohorte de estudiantes.

Con la información de la cohorte Abril - Agosto 2012 se busca caracterizar al abandono, de tal forma que se pueda encontrar el factor que más información contenga en el momento de explicar la permanencia o el abandono.

Explicar el comportamiento de un individuo es una labor compleja ya que cada uno tiene su comportamiento muy particular; para efectos cuantitativos interesa buscar características o determinantes que contengan la mayor cantidad de información posible y que aproximen al verdadero comportamiento del individuo. $\mathrm{Si}$ hacemos correctamente este proceso podremos concretar en un determinante principal del abandono.

La motivación es un factor fundamental que hace que los agentes que conforman el sistema se vuelvan dinámicos y alcancen sus objetivos de corto, mediano y largo plazo. Para el caso de los estudiantes la motivación que define su permanencia en el sistema educativo son los resultados, principalmente los inmediatos, son estos los que lo motivan a continuar, minimizando los otros problemas que se generaron en el proceso de adaptación.

En este escenario, los resultados cuantitativos de la investigación ratifican que existe una íntima relación entre la integración académica de los nuevos estudiantes y la decisión de permanecer o abandonar. Específicamente las estimaciones probabilísticas encuentran una relación negativa entre los resultados promedios y el abandono, por lo tanto a medida que se incrementan los resultados la probabilidad de abandonar disminuye.

\section{TEORÍA DEL ABANDONO}

Un sistema de estudios universitarios a distancia tiene como núcleo al estudiante, al que la universidad le proporciona los recursos institucionales necesarios para facilitar la acción educativa (Rubio Gómez, 2013). Pese a los grandes esfuerzos que realizan los agentes que intervienen en los procesos, siempre existen fenómenos que afectan el normal desenvolvimiento de este bien ordenado sistema.

El abandono ${ }^{1}$ de corto plazo o simplemente el abandono de los estudiantes de nuevo ingreso es uno de los grandes problemas que alteran el comportamiento de los agentes del sistema, es muy conocido que al final del primer ciclo (año o semestre) de estudios es cuando se reduce considerablemente la cohorte de estudiantes 
(Corominas Rovira, 2001). Las causas que generen e impulsan este fenómeno son múltiples con consecuencias directas principalmente en el estudiante.

Las causas y los efectos del abandono han sido ampliamente analizados y a partir de ello se han planteado teorías y modelos que pueden no ser transferibles o aplicables en la realidad de todos los países (Corominas Rovira, 2001), en este caso es necesario realizar un planteamiento para el caso particular del Ecuador. Los estudios realizados por Álvarez Manrique (1997); Bethencour, Cabrera Pérez, Hernández Cabrera, Álvarez Pérez, \& González Afonso (2008); Donoso \& Schiefelbein (2007), presentan un amplio y elaborado marco de análisis de los autores y modelos teóricos del abandono universitario. Por ejemplo, Álvarez Manrique (1997) enriquece el trabajo de Barquero (1993) y realiza una completa clasificación de los determinantes del abandono y los modelos planteados por un gran número de autores; efectúa una división que resulta de nuestro particular interés, porque tácitamente divide al abandono en dos macrocomponentes, los primeros atribuibles a cuestiones personales, familiares y del entorno social y económico y los segundos académicos (bajo rendimiento académico). Asimismo, en el trabajo "Variables psicológicas y educativas en el abandono universitario" de Bethencour, Cabrera Pérez, Hernández Cabrera, Álvarez Pérez, \& González Afonso (2008) se presentan cuatro modelos teóricos (adaptación, estructural, economicista, psicoeducativo) que los autores enmarcan en dos variables: la primera atribuible al alumno y la segunda al contexto académico y social. Con esto podemos afirmar que en el abandono existe una íntima relación entre la parte personal del estudiante y su parte académica o integración académica y social.

La revisión teórica prueba que el abandono es un fenómeno multifactorial (Bethencour, Cabrera Pérez, Hernández Cabrera, Álvarez Pérez, \& González Afonso, 2008), incluso se puede advertir que cada individuo podría presentar causas muy diversas, lo que hace impredecible su comportamiento, limita el análisis y la generación de evidencia. En todo fenómeno siempre hay una característica destacable que brinda luces a las limitaciones citadas, es labor nuestra encontrarla y probar su relevancia.

El elemento común encontrado en los modelos, lo cita Corominas Rovira (2001) al analizar los trabajos de Tinto $(1975,1993)$ y Pascarella y Terenzini (1991), es el nivel de integración académica y social; este es el factor que más influye en el momento de tomar la decisión de permanecer o abandonar los estudios superiores. Asimismo, nos mueve la convicción de que esta integración es la razón general más poderosa, ya que la mayor recompensa para un estudiante son los resultados inmediatos. Los argumentos a favor son varios, se resumen en el hecho de que el estudiante al no tener los resultados esperados se desmotiva porque no cumple con sus expectativas de buen rendimiento académico, en esta circunstancia opta por realizar actividades 
que le generen un mayor grado de satisfacción personal (Donoso \& Schiefelbein, 2007; Bennett, 2003), las pondera excesivamente y les atribuye su salida de la universidad.

El atribuir la salida de la universidad a factores como el trabajo, situación económica, edad, poco tiempo para estar con la familia, etc., no ayuda a clarificar la verdadera razón, es muy difícil que un estudiante acepte que su salida estuvo altamente influenciada por el nivel mostrado o por el escaso nivel de integración académico y social. En estudios realizados a una muestra de estudiantes que abandonan, la falta de tiempo para dedicarle al estudio es el principal argumento, pero indirectamente se ha podido determinar que tiene muchos problemas académicos ya que se encuentra con un sistema de estudios que exige dedicación, actividad, investigación, creatividad, y ordenamiento de la conducta.

\section{Adaptación o nivel de integración académico y social}

El modelo de integración tiene dos componentes, el primero la integración académica siguiendo los planteamientos de Tinto, Donoso \& Schiefelbein (2007) y Cabrera, Bethencourt, Alvarez Pérez, \& González Afonso (2006), quienes señalan que se mide a través de los resultados o calificaciones obtenidas en el ciclo de estudio; y el segundo la integración social que se mide por el número de participaciones o actividades no obligatorias con sus compañeros y profesores. Al respecto de este segundo factor, en educación a distancia se podría iniciar un amplio debate con múltiples argumentos a favor y en contra. En nuestro caso debemos hacer una puntualización, el alumno se encuentra físicamente en diversos lugares (dispersión geográfica), lejos de sus profesores y compañeros, tiene una comunicación no directa, por lo tanto se encuentra estudiando solo (Rubio Gómez, 2011), hay una separación espacial y temporal entre los dos actores, por lo que produce que el estudio a distancia sea mayormente independiente (García Aretio, 2001; Romero Fernández \& Rubio Gómez, 2007). Las facilidades tecnológicas, entornos virtuales, no suplen en su totalidad la deficiencia de la separación, por lo tanto no podemos tener información concreta de su integración social.

En la situación descrita en el párrafo anterior es evidente que el proceso de adaptación de un estudiante de modalidad a distancia está relacionado básicamente a su integración académica y por lo tanto existe una estrecha relación entre el abandono y los resultados inmediatos. Esta afirmación nos permite concluir que la satisfacción con los resultados opera directamente con la intensión de permanecer o abandonar y que los buenos resultados están en relación directa con la permanencia y los malos resultados con el abandono. "De esta forma, si los beneficios de permanecer 
en la institución son percibidos por los estudiantes como mayores que los costos personales, entonces este permanecerá en la institución. Alternativamente, si se reconocen otras actividades como fuentes de mayores recompensas, el estudiante tenderá a abandonar" (Donoso \& Schiefelbein, 2007).

Gráficamente la diferencia básica entre la permanencia y el abandono se presenta en la Figura 1. En el caso de la permanencia, a medida que los resultados tienden a su máximo, la probabilidad de que los estudiantes permanezcan se incrementa hasta llegar a su valor máximo (uno), mientras que la probabilidad de abandonar decrece hasta llegar al mínimo (cero). La curva de abandono tiene un comportamiento contrario a la de permanencia, cuando los resultados inmediatos tienden al mínimo, la probabilidad de abandonar tiende a su máximo y viceversa.

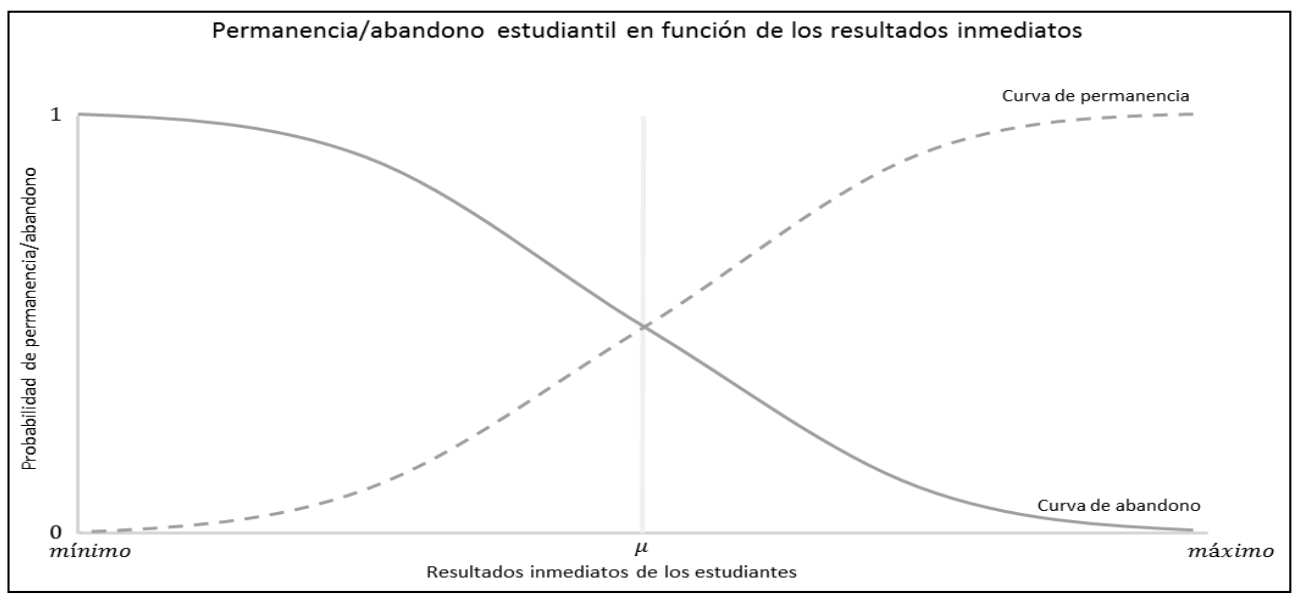

Figura 1. Permanencia/abandono estudiantil en función de los resultados inmediatos

El modelo de adaptación o integración académico contiene la mayor cantidad de información que permita explicar el fenómeno del abandono, pero siempre habrá una parte estocástica que se explique por otros factores. Este planteamiento nos reporta grandes ventajas ya que simplifica el análisis y permite utilizar información cuantitativa de los resultados inmediatos de los estudiantes.

\section{Caracterización del abandono en función de los resultados inmediatos}

La teoría del abandono basada en el modelo de integración académica permite predecir el comportamiento que tendrán las curvas de permanencia y abandono, en cada punto de estas curvas se relaciona un resultado inmediato promedio con el número de estudiantes de la población que están en esa condición. 
En la Figura 2 se representa un escenario característico de las dos curvas, las condiciones teóricas para su planteamiento son:

La recta $A A^{\prime}$ es la línea de los resultados mínimo y máximo, en esta recta siempre hay un punto mínimo de buen rendimiento (B) el que lo establece o fija la universidad, desde este punto hacia la derecha se considera el buen rendimiento y la izquierda el mal rendimiento. El punto medio de rendimiento $\left(\mu=\frac{A+A^{\prime}}{2}\right)$ se ubica en la mitad de la recta de resultados.

Por la forma de establecer el punto medio (), el mínimo de buen rendimiento (B) es superior a este valor:

$$
B>\mu
$$

La condición $B>\mu$ establece que la exigencia de la Universidad es superior al promedio de los resultados de todos los individuos. La brecha que se genera repercute en el abandono, si se cumple este supuesto los estudiantes no tienen la disposición o los conocimientos necesarios ya que no llegan al mínimo de buen rendimiento exigido por la Universidad.

La permanencia está en relación directa con los buenos resultados $(\geq B)$, por lo tanto se espera que la mayoría de estudiantes que superan el punto mínimo de buen rendimiento (B) se mantengan activos, el número de estudiantes en esta condición irá decreciendo a medida que los resultados se acerquen a su máximo.

El mayor número de estudiantes que abandonan se ubicarán a la izquierda del punto mínimo de buen rendimiento (B) distribuidos normalmente en torno a la media $(\mu)$ de los resultados, se asume la normalidad por el tamaño de la población que se involucra en el estudio. 


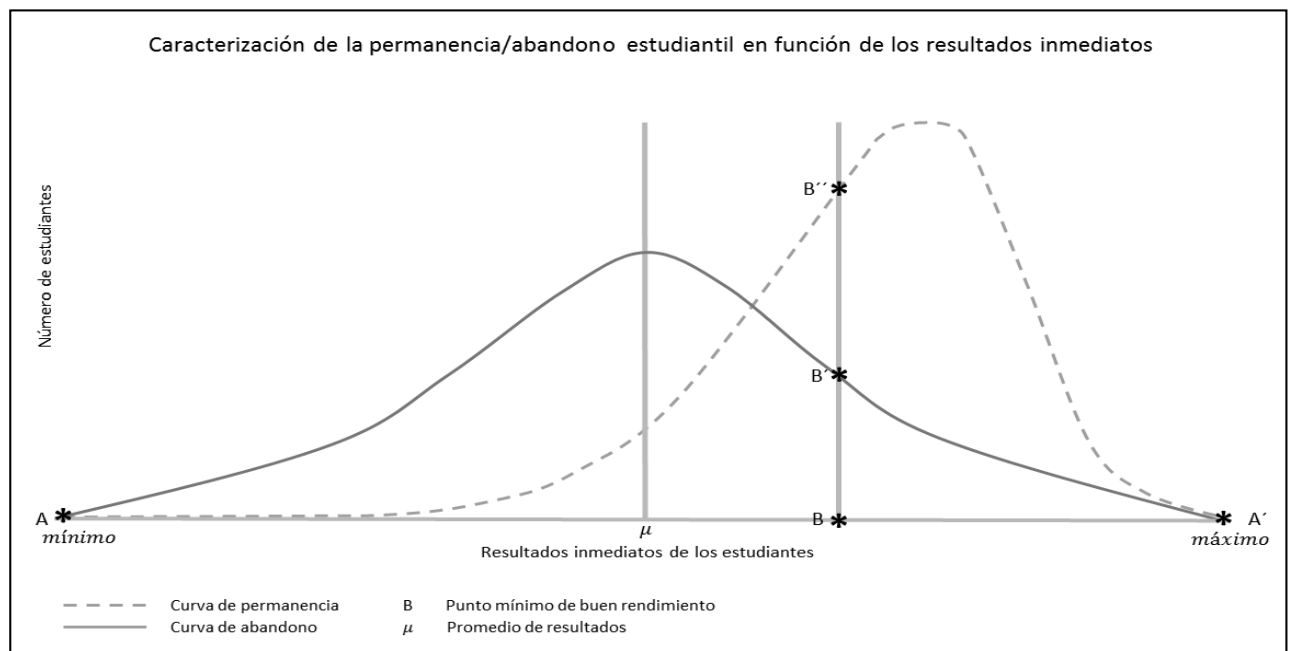

Figura 2. Caracterización de la permanencia/abandono estudiantil en función de los resultados inmediatos

La caracterización descrita y presentada en la Figura 2 permite agrupar la información de la decisión que toman los estudiantes al finalizar el primer ciclo de estudios.

La región comprendida entre la media de rendimiento $(\mu)$ y el punto mínimo de buen rendimiento (B) es importante porque es ahí donde se cruzan las dos curvas, los estudiantes que están en el punto de cruce, el cincuenta por ciento abandonan y el cincuenta por ciento permanecen, es a partir de ahí que la motivación por la permanencia crece y la del abandono decrece, por lo tanto el desplazamiento positivo de la media de rendimiento o la convergencia al mínimo de buen rendimiento, hará que más estudiantes se sientan motivados por permanecer.

La prolongación del punto mínimo de buen rendimiento (BB'B' ') divide las dos curvas en cuatro zonas generales:

\begin{tabular}{|lll|}
\hline ZONA & TIPO & DESCRIPCIÓN \\
\hline AB $^{\prime}$ & $\begin{array}{l}\text { Abandono por } \\
\text { motivos académicos. }\end{array}$ & $\begin{array}{l}\text { Estudiantes que no alcanzan el punto mínimo de buen } \\
\text { rendimiento y no están motivados a continuar. Cubre la } \\
\text { mayor parte de la curva. }\end{array}$ \\
\hline B $^{\prime} \mathbf{A}^{\prime}$ & $\begin{array}{l}\text { Abandono por } \\
\text { motivos no } \\
\text { académicos. }\end{array}$ & $\begin{array}{l}\text { Estudiantes que se ven afectados por factores exógenos. } \\
\text { Las razones se las podría catalogar como variables no } \\
\text { observables. }\end{array}$ \\
\hline
\end{tabular}




\begin{tabular}{|lll|}
\hline ZONA & TIPO & DESCRIPCIÓN \\
\hline B' A' $^{\prime}$ & $\begin{array}{l}\text { Permanencia por } \\
\text { motivos académicos. }\end{array}$ & $\begin{array}{l}\text { Estudiantes que tienen motivación por los resultados. } \\
\text { Cubre la mayor parte de la curva. }\end{array}$ \\
\hline AB $^{\prime \prime}$ & $\begin{array}{l}\text { Permanencia } \\
\text { por motivos no } \\
\text { académicos. }\end{array}$ & Estudiantes que tienen otro tipo de motivación. \\
\hline
\end{tabular}

Cuadro 1. Zonas en las que se dividen las curvas de abandono y permanencia

Las expectativas de las proporciones de cada zona o área se crean considerando que la permanencia es igual al abandono y que los resultados ejercen una influencia directa en la motivación y posterior decisión de los estudiantes. Con estas condiciones se espera que el abandono por motivos académicos sea mayor que el abandono por motivos no académicos $\left(\mathrm{AB}^{\prime}>\mathrm{B}^{\prime} \mathrm{A}^{\prime}\right)$ y que la permanencia por motivos académicos sea mayor a la permanencia por motivos no académicos $\left(\mathrm{B}^{\prime \prime} \mathrm{A}^{\prime}>\mathrm{AB}^{\prime \prime}\right)$.

Caracterizar el fenómeno vuelve práctico el análisis ya que agrupa a los individuos bajo una característica común, elimina muchos de los distractores o características individuales de los estudiantes. Las poblaciones numerosas son difíciles de estudiar y especificar una muestra, sin tener información a priori, puede sesgar los resultados, la caracterización permite enfocar las investigaciones y tener resultados más eficientes.

Probar esta parte de la teoría del abandono, en diferentes escenarios y con agentes del sistema académico con características heterogéneas le dará la solidez necesaria para confirmar que la integración académica es la causa principal, no la única, del abandono.

Es innegable que los resultados no son la única causa del abandono, siempre habrá factores exógenos que influyan directa o indirectamente. El abandono y la permanencia por motivos no académicos $\left(\mathrm{B}^{\prime} \mathrm{A}^{\prime}\right.$ y $\left.\mathrm{AB}^{\prime \prime}\right)$ son una muestra de que existen otros factores, en estos casos los estudiantes toman la decisión contraria a lo que se esperaría en función a sus resultados, ellos tienen un tipo diferente y particular de motivación. La evidencia probará que estas son las áreas con menor cantidad de estudiantes y también confirmará la teoría general de que existe una relación negativa entre el abandono y la integración académica o resultados. 


\section{LOS NUEVOS ESTUDIANTES MATRICULADOS EN UN SISTEMA DE ESTUDIOS SUPERIOR A DISTANCIA EN EL ECUADOR. ESCENARIO DE PRUEBA DE LA TEORÍA}

El sistema de educación superior a distancia agrupa a miles de personas en el Ecuador y el mundo; con cada ciclo de estudio el número crece exponencialmente, esto se debe a que en este sistema de estudios las personas han encontrado la forma idónea de cumplir con su meta de profesionalización (Rubio Gómez, 2011), independientemente de los factores de identificación, académicos, psicológicos y sociofamiliares.

En Ecuador, el crecimiento de la población estudiantil universitaria matriculada en el sistema de estudios a distancia, sobre todo los de nuevo ingreso, está en el orden del 10\% anual y según informe de la Secretaría Nacional de Educación Superior, Ciencia, Tecnología e Innovación (SENESCYT) la Universidad Técnica Particular de Loja (UTPL) concentra al 82\% de la población de nuevos estudiantes y al 83\% de la población total ${ }^{2}$.

Las características que se describirán corresponden a 6.751 estudiantes matriculados en la cohorte abril - agosto 2012 de la Universidad Técnica Particular de Loja. Esta población está distribuida en todo el territorio nacional ecuatoriano, a través de la red de centros universitarios y oficinas de información y gestión, en 82 ciudades, cantones y parroquias de las 24 provincias del país, también en tres centros universitarios en el extranjero.

Al tomar esta base de información aseguramos que los resultados son trasladables al comportamiento de la población estudiantil de nuevo ingreso en un sistema de educación superior a distancia del Ecuador.

\section{Características de la población analizada}

Las características de la población se desagregan en tres: identificación, académicas y abandono.

\section{Identificación}

Las variables de identificación permiten delinear el perfil del estudiante, que en el caso del sistema de estudios a distancia es muy heterogéneo. La edad, sexo y sector geográfico son las tres variables de identificación utilizadas. 
La cohorte de estudiantes es mayoritariamente femenina (61\%) y vive en centros urbanos, principalmente en las ciudades más pobladas del país. En cuanto a la edad se presenta la mayor dispersión, con un mínimo de 17 años y un máximo de 71 años, tiene una moda de edad de 20 años y un promedio de 28 años.

Los resultados de la cohorte se presentan en la siguiente tabla:

\begin{tabular}{|c|c|c|c|c|c|c|}
\hline \multirow{2}{*}{$\begin{array}{c}\text { RANGO } \\
\text { EDAD } \\
\text { AÑOS }\end{array}$} & \multicolumn{2}{|c|}{ URBANO } & \multirow{2}{*}{ URBANO } & \multicolumn{2}{|c|}{ RURAL } & \multirow{2}{*}{ RURAL } \\
\hline & MUJERES & HOMBRES & & MUJERES & HOMBRES & \\
\hline 17 A 19 & $6 \%$ & $3 \%$ & $5 \%$ & $17 \%$ & $10 \%$ & $15 \%$ \\
\hline 20 A 25 & $45 \%$ & $37 \%$ & $42 \%$ & $47 \%$ & $45 \%$ & $46 \%$ \\
\hline $26 \mathrm{~A} 30$ & $21 \%$ & $25 \%$ & $23 \%$ & $18 \%$ & $19 \%$ & $19 \%$ \\
\hline 31 A 35 & $14 \%$ & $15 \%$ & $14 \%$ & $10 \%$ & $14 \%$ & $11 \%$ \\
\hline $36 \mathrm{~A} 40$ & $7 \%$ & $10 \%$ & $8 \%$ & $4 \%$ & $6 \%$ & $4 \%$ \\
\hline $41 \mathrm{~A} 45$ & $4 \%$ & $5 \%$ & $4 \%$ & $2 \%$ & $4 \%$ & $2 \%$ \\
\hline $46 \mathrm{~A} 50$ & $2 \%$ & $3 \%$ & $3 \%$ & $1 \%$ & $1 \%$ & $1 \%$ \\
\hline $51 \mathrm{~A} 55$ & $1 \%$ & $1 \%$ & $1 \%$ & $1 \%$ & $1 \%$ & $1 \%$ \\
\hline MÁS DE 56 & $1 \%$ & $0 \%$ & $1 \%$ & O\% & O\% & O\% \\
\hline TOTAL & $100 \%$ & $100 \%$ & $100 \%$ & $100 \%$ & $100 \%$ & $100 \%$ \\
\hline
\end{tabular}

Tabla 1. Características de identificación de la cohorte de estudiantes

La tabla 1 evidencia una población muy diversa, con ello el sistema de estudios a distancia cumple con el principio de democratizar la educación, la integración al estudio universitario de diversos grupos de personas es una característica propia del sistema de estudios a distancia. Algunas de estas particularidades son:

- La población urbana y rural, se concentra en el grupo de edad de 20 a 25 años, mayoritariamente mujeres, esta diferencia es más notoria en el sector urbano.

- La mayor proporción de mujeres (80\%) del sector urbano está entre 20 a 35 años, mientras que en el sector rural (82\%) está entre 17 a 30 años.

- En el caso de los hombres del sector urbano y rural, en las edades comprendidas entre 20 a 35 años, se tienen el $77 \%$ y $78 \%$ respectivamente.

- En el sector rural se presenta una particularidad en el rango de menores de 20 años, las mujeres son una proporción significativa del total de la población 
de este sector. Este es un indicador del nivel de inclusión que tiene el sistema de estudios a distancia.

- No existen estudiantes del sector rural que superen los 55 años, y en el caso del sector urbano de esta categoría de edad solo se han matriculado mujeres.

El retraso en la incorporación a los estudios es la cantidad de años que el estudiante tarde en matricularse en la Universidad, se mide desde los diecisiete ${ }^{3}$ años hasta la edad (años) que el estudiante tiene en el momento de realizar su matrícula, los resultados se exponen en la Figura 3.

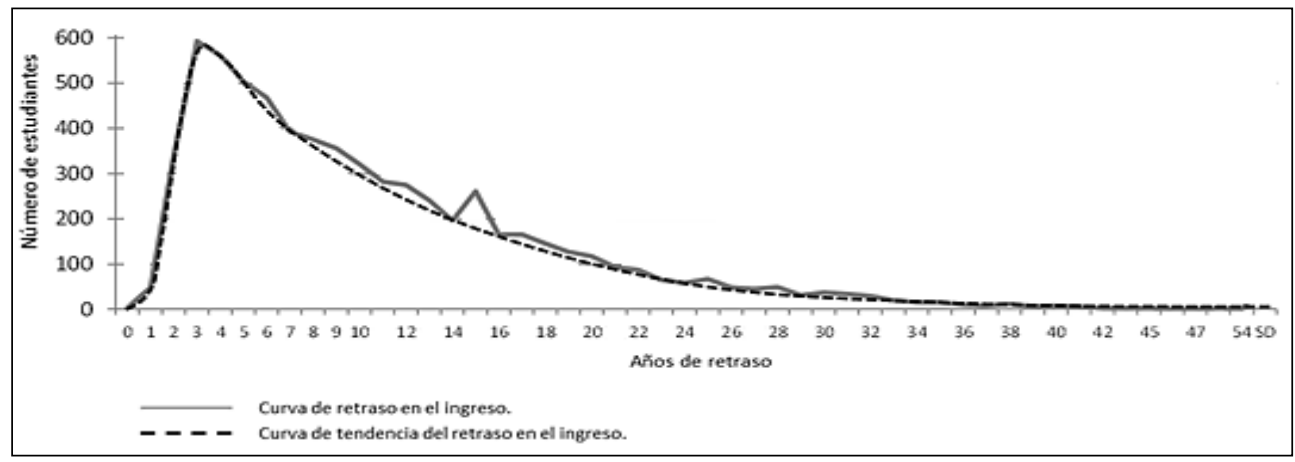

Figura 3. Retraso en la incorporación a los estudios

En la cohorte de estudiantes seleccionada la mayoría han postergado su ingreso en al menos tres años, la relación años de retraso con el rendimiento académico es negativa, en general se espera que el estudiante más joven tenga un mejor rendimiento. En este caso los años de retraso los tomamos como una característica de identificación de los estudiantes y también prueba la diversidad de perfiles de los estudiantes.

\section{Resultados académicos}

Las características académicas más relevantes de la cohorte de estudiantes se recogen en la Tabla 2. Trabajar con datos de estudiantes que pertenecen a diferentes áreas académicas no resulta un problema porque todos ellos toman inicialmente tres asignaturas de formación básica, esta característica vuelve a la población homogénea en sus inicios. 


\begin{tabular}{|c|c|c|c|c|c|}
\hline ÁREA & 岁 & 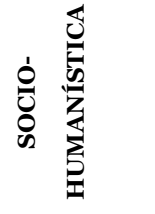 & $\begin{array}{l}\text { త్ర } \\
0 \\
0 \\
0 \\
0 \\
0\end{array}$ & 岁 & TOTAL \\
\hline $\mathrm{N}^{\circ}$ DE TITULACIONES & 10 & 7 & 1 & 1 & 19 \\
\hline $\mathrm{N}^{\circ}$ ESTUDIANTES & 2612 & 3209 & 561 & 369 & 6751 \\
\hline $\begin{array}{l}\text { PORCENTAJE DE } \\
\text { ESTUDIANTES }\end{array}$ & $39 \%$ & $48 \%$ & $8 \%$ & $5 \%$ & $100 \%$ \\
\hline $\begin{array}{l}\text { ASIGNATURAS } \\
\text { FORMACIÓN BÁSICA }\end{array}$ & 3 & 3 & 3 & 3 & 3 \\
\hline OTROS ASIGNATURAS & 2 & 2 & 2 & 2 & 2 \\
\hline TOTAL ASIGNATURAS & 4 & 4 & 5 & 4 & 4 \\
\hline de RAZÓN ${ }^{4}$ & 0,4 & 0,5 & 0,5 & 0,4 & 0,5 \\
\hline
\end{tabular}

Tabla 2. Características académicas de la cohorte de estudiantes

Cuatro grandes áreas académicas son ofertadas, cada una de ellas tienen sus propias titulaciones ${ }^{5}$, el área administrativa es la que mayor oferta tiene, diez titulaciones, pero el área sociohumanística es la que mayor demanda tiene en sus siete titulaciones. En cuanto al número de asignaturas matriculadas no se observan diferencias significativas, solo el hecho de que en promedio están aprobando el $50 \%$ de las asignaturas matriculadas, el $40 \%$ en las áreas administrativa y técnica y el 50\% en las áreas sociohumanística y biológica; esto último es una medida de la baja eficiencia que tiene esta cohorte de estudiantes y que incide en la decisión de permanecer o abandonar.

El nivel de aprobación/reprobación es una característica fundamental ya que representa el nivel de adaptación o integración académica del estudiante. La figura 4 presenta los resultados relativos generales, el 52\% del total de asignaturas matriculados se han reprobado, el $47 \%$ son aprobados, por lo que ratifica la medida (razón) de eficiencia expuesta en la Tabla 2. 


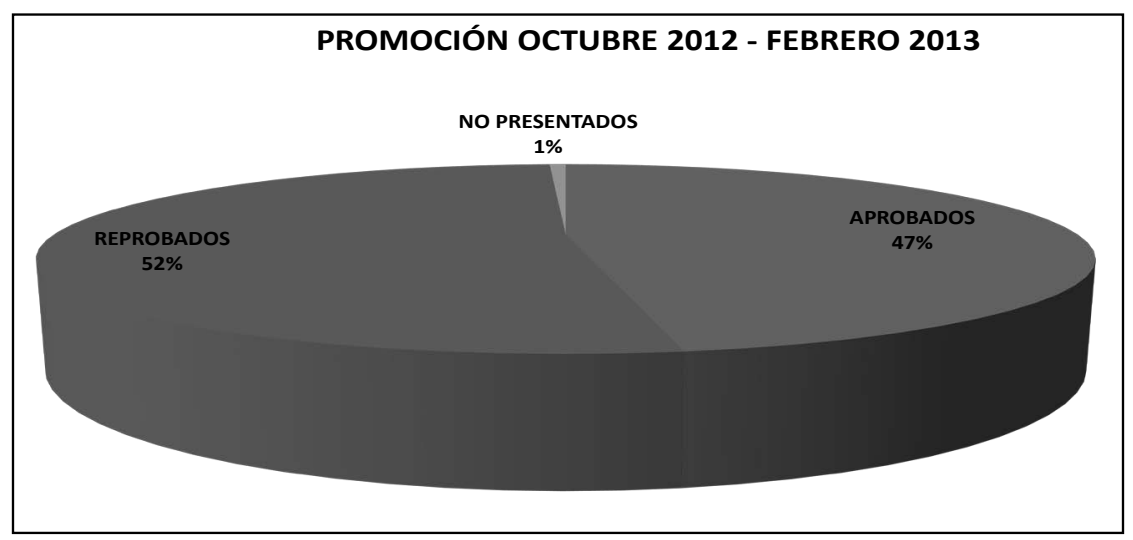

Figura 4. Resultados de acuerdo a las asignaturas matriculados

Siguiendo la teoría que señala que la permanencia/abandono está en relación con los resultados, se espera que la permanencia/abandono tenga proporciones similares, es decir que esté alrededor del 50\% de los estudiantes.

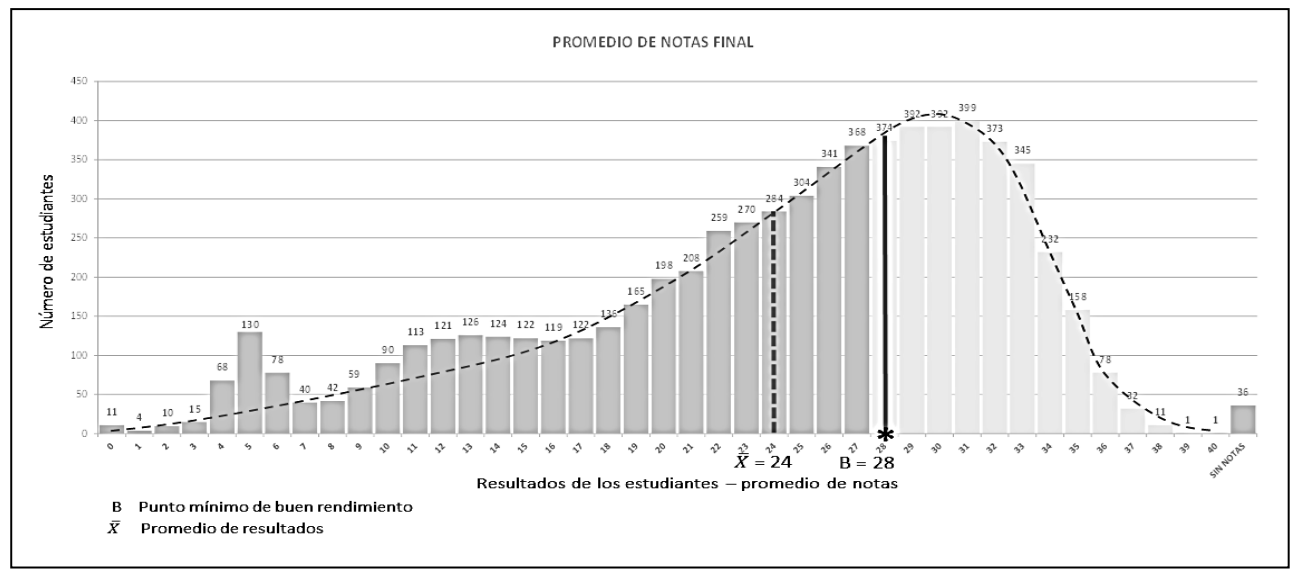

Figura 5. Promedio de notas finales

El histograma de la figura 5 muestra la distribución de los resultados promedios de cada uno de los estudiantes, tres son sus características: primera, la escala de valoración tiene un mínimo de cero y un máximo de cuarenta puntos, segunda el valor mínimo de buen rendimiento es 28 puntos $^{6}$, y tercera la media general de los resultados es igual a 24 puntos.

La forma suavizada de los resultados es el comportamiento hipotético que se asumió para la caracterización de la permanencia, se espera que la permanencia real siga esta figura. 
Los resultados no se distribuyen alrededor de la media, por lo tanto no tienen un comportamiento normal, están sesgados al punto mínimo de buen rendimiento. Son muy pocos los estudiantes que tienen un rendimiento sobresaliente ${ }^{7}(0,2 \%)$ igual o mayor a treinta y ocho puntos, por el contrario sí hay muchos estudiantes que tienen un rendimiento muy bajo $(8,1 \%)$ igual o menor a diez puntos.

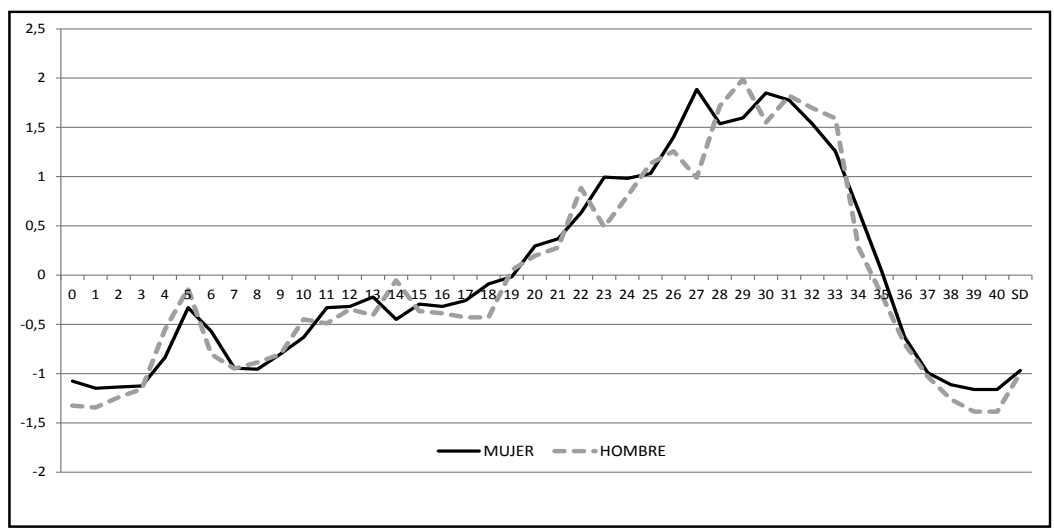

(a)

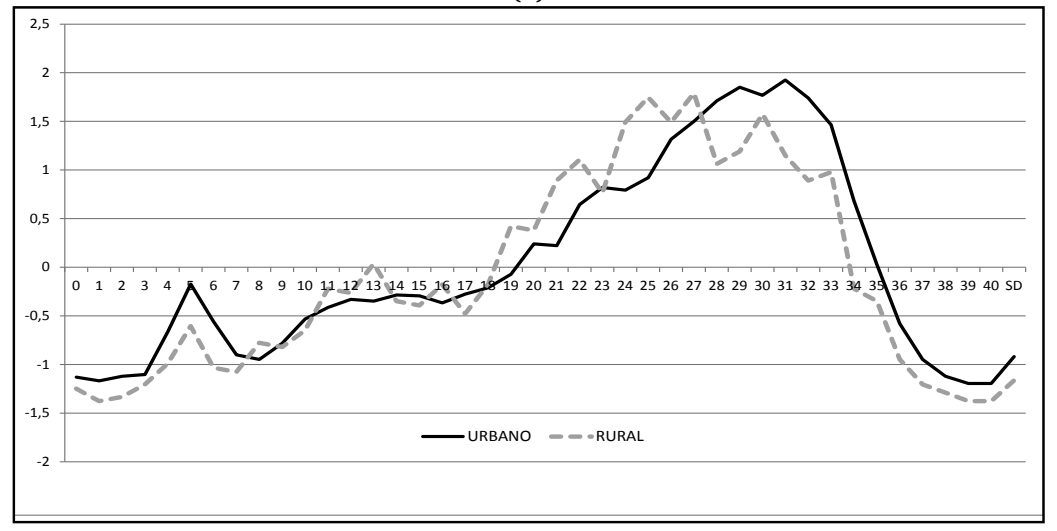

(b)

Figura 6. Resultados estandarizados

En la figura 6 (a) y (b) se presentan los resultados estandarizados de las variables sexo y región geográfica comparados con los resultados promedios. El comportamiento general en los dos casos es similar en cuanto a la concentración de la información, específicamente la figura 6 (a) no muestra diferencias entre los resultados de mujeres y hombres, en cambio que sí se aprecia diferencias entre los resultados del sector, figura 6 (b), los estudiantes que viven en el sector rural, que en proporción son el 19\%, tienen una concentración muy cercana a la media 24 puntos, 
mientras que los del sector urbano están en torno a los 28 puntos; estos resultados se explican porque uno de los determinantes principales del rendimiento académico es el sector en el que habitan los estudiantes (Moncada \& Rubio, 2011). Las variables sexo y sector, así como la edad y el número de asignaturas matriculadas, son determinantes de los resultados académicos inmediatos, está probada su influencia, en esta investigación no se realizan pruebas al respecto.

\section{Característica del abandono en el Ecuador}

En el Ecuador el abandono de corto plazo es un fenómeno que afecta a una enorme proporción de la población estudiantil: todos los semestres son miles los estudiantes que se encuentran en la disyuntiva de permanecer o abandonar. En proporción, desde el 2004 hasta el 2012 en la Universidad Técnica Particular de Loja el comportamiento ha sido el mismo figura 7: las cifras señalan que el $50 \%$ de los estudiantes toman la decisión de abandonar.

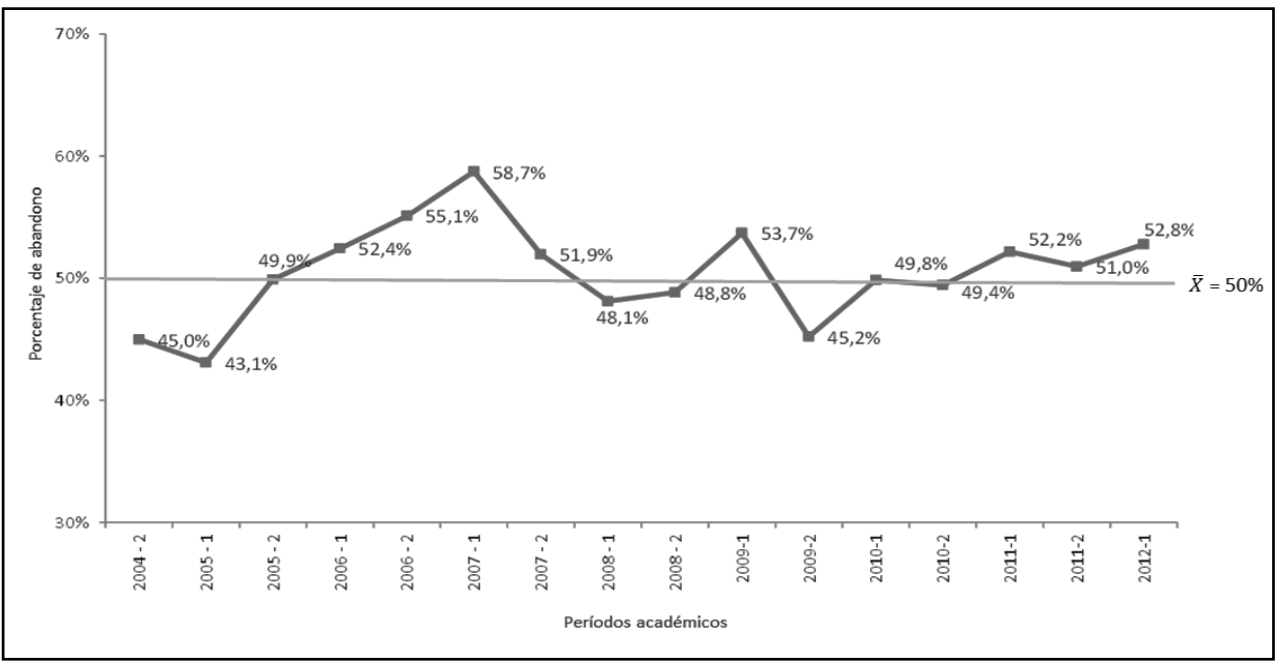

Figura 7. Abandono estudiantil en el corto plazo

El comportamiento semestral del abandono varía en torno a su media: se observan pocos puntos diferentes que no tienen una explicación de fondo o un factor exógeno que haya influido, los cambios son atribuibles a las características propias de las cohortes de estudiantes. Los cambios institucionales y los que progresivamente ha implementado el Estado no han ayudado en el aumento de la tasa de permanencia, el abandono persiste, esto convierte al promedio del abandono de estos años en la tasa natural de abandono estudiantil en el corto plazo. 
La situación del alto abandono en el Ecuador, independiente de la modalidad de estudio, no es una condición diferente a la que tienen muchos de los países de América, este comportamiento se repite y en algunos casos como Brasil, Uruguay, Bolivia y República Dominicana es más agudo. Kuna, García-Martínez, \& Villatoro (s.f) en el trabajo "Identificación de Causales de Abandono de Estudios Universitarios. Uso de Procesos de Explotación de Información” exponen el porcentaje de abandono general en once países americanos, en promedio 54\%, no presentan información para el caso ecuatoriano pero devela esta realidad en la que, con los resultados que vamos obteniendo, todos estamos incluidos.

\section{Caracterización del abandono en función de los resultados inmediatos en el Ecuador}

La teoría relaciona directamente a la permanencia/abandono con los resultados, como medida inmediata del nivel de adaptación o integración académica del estudiante. Una forma de generar evidencia es aplicar la caracterización de la figura 2 para los datos de la cohorte de estudiantes del 2012 en el Ecuador. La variable principal se obtuvo al verificar si el estudiante tiene matrícula en el periodo inmediatamente posterior, es decir si está o no en los registros de Octubre 2012 Febrero 2013. Los resultados producto de la verificación dan lugar a las curvas que se presentan en la Figura 8 (a).

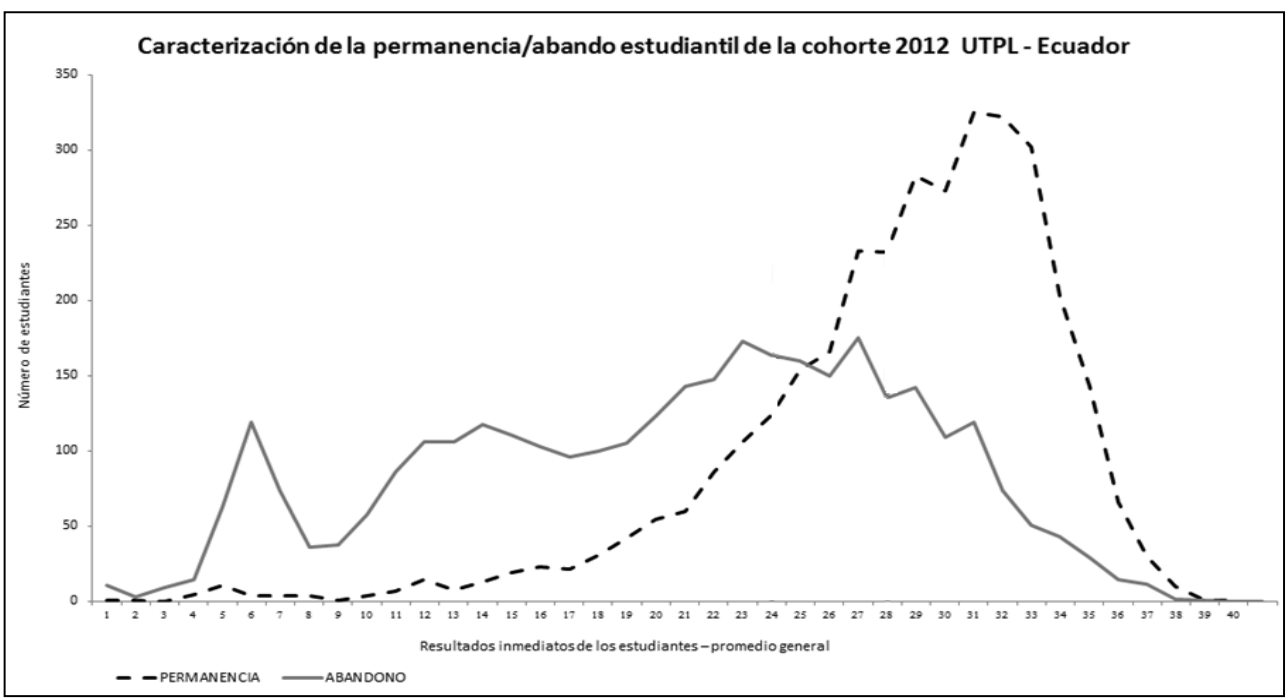

Figura 8 (a). Caracterización de la permanencia/abandono estudiantil de la cohorte 2012 UTPL Ecuador 
Las curvas de permanencia y abandono en el Ecuador, figura 8 (a) tienen el comportamiento esperado y cumplen con las expectativas descritas en la caracterización teórica. La permanencia tiene sesgo importante y vemos como aumenta a medida que los resultados tienden a su valor máximo, en cambio el abandono se distribuye con mayor normalidad en torno a los 24 puntos de media.

En la figura 8 (b) se suavizan las curvas de la figura 8 (a), eliminando aquellos puntos que puedan resultar de un comportamiento atípico.

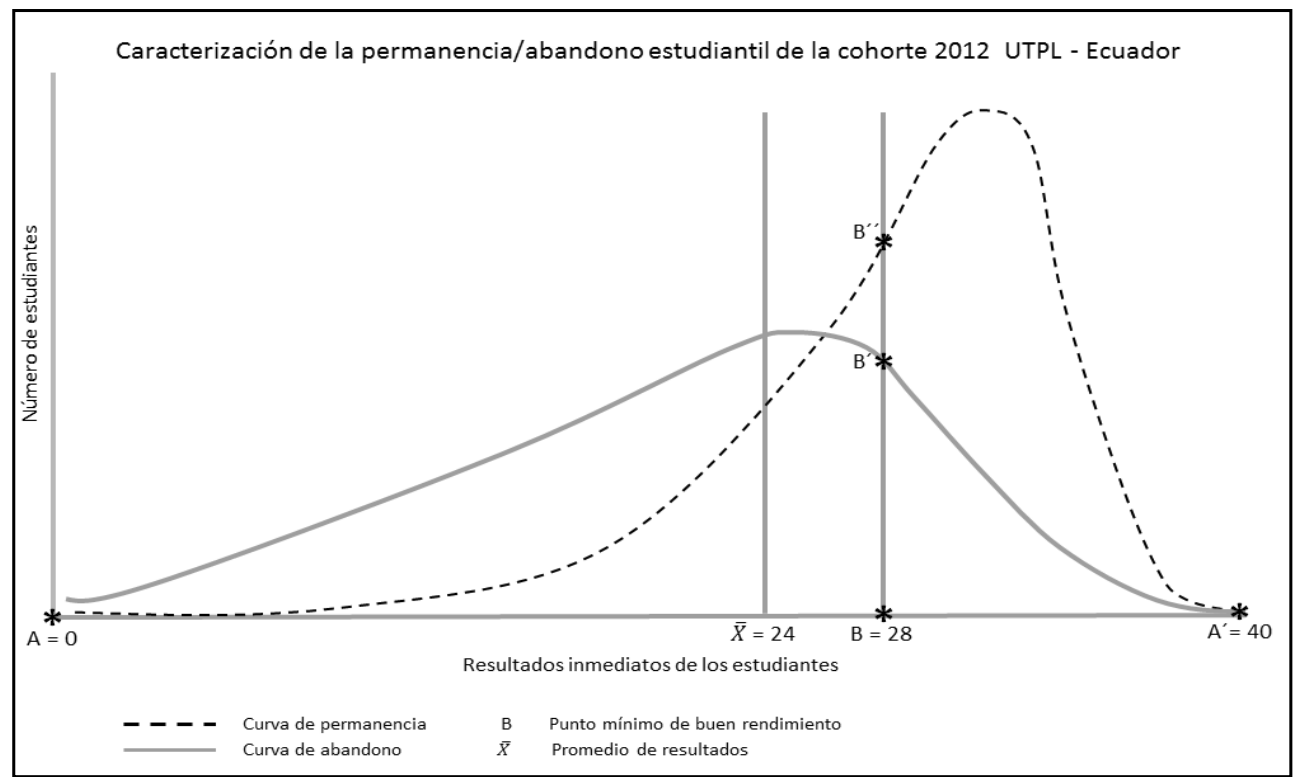

Figura 8 (b). Caracterización de la permanencia/abandono estudiantil de la cohorte 2012 UTPL Ecuador

Las expectativas que se plantearon en la caracterización teórica se ven reflejadas en la figura 8 (b). El mayor número de estudiantes que decide abandonar se encuentra antes de los 28 puntos (B) y la mayoría de los que deciden permanecer posterior a este punto. Los resultados de cada una las áreas en la que la caracterización divide a las curvas de abandono y permanencia están en el cuadro 2.

\begin{tabular}{|l|l|c|c|}
\hline ZONA & \multicolumn{1}{|c|}{ TIPO } & $\begin{array}{c}\text { ÁREA } \\
\text { ESPECÍFICA* }\end{array}$ & $\begin{array}{c}\text { ÁREA } \\
\text { TOTAL** }^{*}\end{array}$ \\
\hline AB $^{\prime}$ & Abandono por motivos académicos. & $82 \%$ & $40,6 \%$ \\
\hline $\mathbf{B}^{\prime} \mathbf{A}^{\prime}$ & Abandono por motivos no académicos. & $\mathbf{1 8 \%}$ & $\mathbf{8 , 9 \%}$ \\
\hline & SUBTOTAL ABANDONO & $\mathbf{1 0 0 \%}$ & $\mathbf{4 9 , 5 \%}$ \\
\hline
\end{tabular}




\begin{tabular}{|l|l|c|c|}
\hline ZONA & \multicolumn{1}{|c|}{ TIPO } & $\begin{array}{c}\text { ÁREA } \\
\text { ESPECÍFICA* }\end{array}$ & $\begin{array}{c}\text { ÁREA } \\
\text { TOTAL** }\end{array}$ \\
\hline B '“A' $^{\prime}$ & Permanencia por motivos académicos. & $65 \%$ & $32,6 \%$ \\
\hline AB' $^{\prime \prime}$ & Permanencia por motivos no académicos. & $35 \%$ & $17,9 \%$ \\
\hline & SUBTOTAL PERMANENCIA & $\mathbf{1 0 0 \%}$ & $\mathbf{5 0 , 5}$ \\
\hline
\end{tabular}

* Proporción tomada de acuerdo al total de cada condición abandono o permanencia. ** Proporción tomada del total de estudiantes.

Cuadro 2. Resultados de las zonas en las curvas de abandono y permanencia

Las áreas específicas del abandono son $\mathrm{AB}^{\prime} \mathrm{y} \mathrm{B}^{\prime} \mathrm{A}^{\prime}$, figura 8 (b), la primera considera que el abandono se da por motivos académicos y es igual al $82 \%$ de los estudiantes que abandonan, y la segunda por motivos no académicos es el $18 \%$. La diferencia entre una y otra categoría confirma que los estudiantes que no alcanzaron el rendimiento mínimo establecido no se sienten estimulados a permanecer y toman la decisión de abandonar, ponderando otras actividades que emocionalmente le generen un mayor grado de satisfacción. También hay estudiantes que alcanzan un buen rendimiento y deciden abandonar, en esos casos sí existen factores diferentes al académico que los obligan a dejar sus estudios recién iniciados.

Las dos áreas de la permanencia son B" 'A' y AB" ', figura 8 (b). El 65\% de la curva la ocupan los estudiantes que deciden permanecer porque sus resultados fueron los deseables y esto los motiva a persistir, mientras que el $35 \%$ deciden continuar pese a que los resultados no fueron los deseables.

El área total que ocupa cada tipo de abandono/permanencia se encuentra dividiendo el número de estudiantes en esta condición para el total de la cohorte. En el resultado general, el abandono y la permanencia son $50 \%$ respectivamente; de los resultados de cada área se puede destacar que el abandono por motivos académicos es el 40,6\% y la permanencia por este mismo motivo es 32,6 \%, confirmando los efectos negativos y positivos que tienen los resultados en el abandono y permanencia.

La condición que el punto mínimo de buen rendimiento es mayor que la media de rendimiento se cumple, en este caso el abandono se produce porque los estudiantes tienen una baja disposición para mejorar sus resultados o no tienen los conocimientos necesarios para cumplir con la exigencia de la Universidad.

Estimación de la probabilidad de permanecer o abandonar

La probabilidad de permanencia/abandono que tienen los estudiantes de la cohorte 2012 se presenta en la figura 9, este gráfico simplifica la perspectiva del 
problema y confirma que los resultados afectan directamente a la probabilidad de permanencia y abandono. El cruce de las curvas coincide en el $50 \%$ de probabilidad y se produce en los 26 puntos de la recta, que es el punto intermedio entre el promedio de calificaciones y el punto mínimo de buen rendimiento, en general este zona puede marcar la indecisión que tendrían los estudiantes entre permanecer o abandonar.

La curva de permanencia avanza positivamente a medida que los resultados tienden a 40 puntos, los estudiantes cuyo promedio es sobresaliente tienen de probabilidad uno (100\%) de permanecer; lo contrario sucede con la curva de abandono: a medida que los resultados tienden al mínimo la probabilidad de abandonar tiende a uno (100\%).

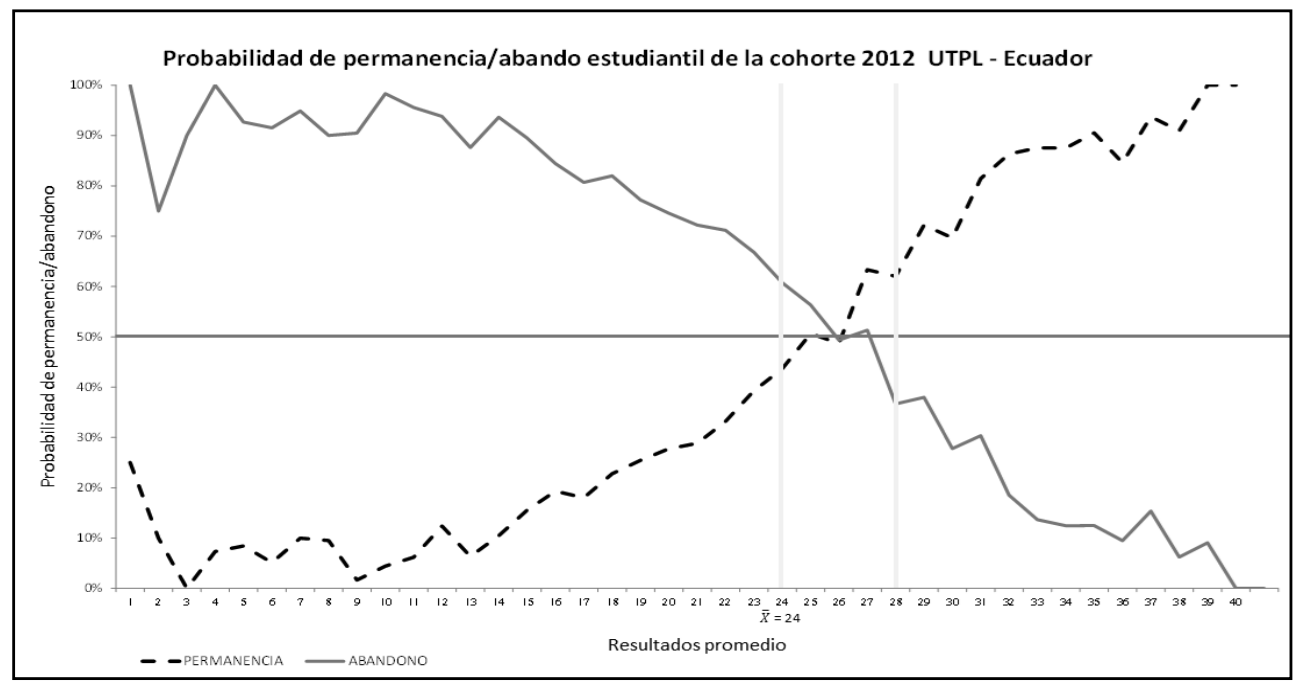

Figura 9. Probabilidad de permanencia/abandono estudiantil de la cohorte 2012 UTPL - Ecuador

Los efectos descritos coinciden con los resultados de las estimaciones realizadas para probarlos (tabla 3).

Se plantea la relación simple entre el abandono $\left(Y_{i}\right)$ y los resultados promedio de cada estudiante o rendimiento académico $\left(X_{i}\right)$. Asimismo por la evidencia generada por Moncada \& Rubio (2011) en la que se demuestra que el rendimiento académico inmediato depende de la edad $\left(Z_{1}\right)$, el sexo $\left(Z_{2}\right)$, los ingresos $\left(Z_{3}\right)$, el número de créditos matriculados $\left(Z_{4}\right)$, la región geográfica $\left(Z_{5}\right)$ y la metodología de estudio $\left(Z_{6}\right)$, por lo tanto estas variables no deben volverse a incluir en el modelo abandono en función del rendimiento académico. 


$$
\begin{gathered}
X_{i}=f\left(Z_{1}, Z_{2}, Z_{3}, Z_{4}, Z_{5}, Z_{6}\right) \\
Y_{i}=f\left(X_{i}\right) \\
P_{i}=E\left(Y_{i}=1 \mid X\right)=\varphi_{1}+\delta_{1} X_{i}+\mu_{i}
\end{gathered}
$$

$\mathrm{Pi}=\mathrm{Yi}=$ Permanencia $\mathrm{o}$ abandono de cada estudiante

$1=$ abandona

$\mathrm{O}=$ permanece

$\mathrm{Xi}=$ resultados promedio de cada estudiante

$\mu \mathrm{i}=$ variable estocástica

\begin{tabular}{|l|l|l|l|l|l|l|}
\hline$P_{\mathrm{i}}=Y_{\mathrm{i}}$ & Coeficiente & Error & $\mathrm{t} / \mathrm{z}$ & $\mathrm{P}>|\mathrm{t} / \mathrm{z}|$ & \multicolumn{1}{|c|}{ Intervalo al 95\% } \\
\hline \multicolumn{7}{|c|}{ MODELO LINEAL DE PROBABILIDAD } \\
\hline$\delta_{1}$ & $-0,033$ & 0,0006 & $-52,71$ & 0,000 & $-0,035$ & $-0,032$ \\
\hline$\varphi_{1}$ & 1,30 & 0,0160 & 80,80 & 0,000 & 1,260 & 1,328 \\
\hline \multicolumn{7}{|c|}{ MODELO LOGIT } \\
\hline$\delta_{1}$ & $-0,192$ & 0,005 & $-37,48$ & 0,000 & $-0,202$ & $-0,182$ \\
\hline$\varphi_{1}$ & 4,741 & 0,134 & 35,29 & 0,000 & 4,478 & 5,005 \\
\hline \multicolumn{7}{|c|}{ MODELO PROBIT } \\
\hline$\delta_{1}$ & $-0,111$ & 0,0027 & $-41,30$ & 0,000 & $-0,116$ & $-0,105$ \\
\hline$\varphi_{1}$ & 2,709 & 0,0700 & 38,83 & 0,000 & 2,572 & 2,845 \\
\hline
\end{tabular}

El efecto negativo de la pendiente coincide en las estimaciones, es decir a medida que se incrementan los resultados promedios finales, la probabilidad de que el estudiante abandone disminuye en determinada proporción. En este caso la proporción (4\% aproximadamente) no es lo más importante, la confirmación del efecto sí, ya que concuerda con lo expuesto en la teoría y la descripción de los datos estadísticos.

Existen muchas variables que se involucran en el momento de tomar una decisión, en este caso permanecer o abandonar los estudios, la figura 9 y los resultados de la estimaciones son una muestra más que los resultados inmediatos o promedio de notas contienen una gran cantidad de información que explica la decisión que toman los estudiante sobre la permanencia o el abandono de los estudios universitarios. 


\section{CONCLUSIONES}

El abandono de corto plazo o simplemente el abandono es un fenómeno de múltiples aristas que afecta principalmente al núcleo del sistema, es decir a los propios estudiantes, pero deja grandes secuelas en la universidad, en el sistema de estudios y en el marco estructural y organizativo de la educación superior. Al tener tantas causas resulta difícil concentrar la atención en la más significativa, muchas de ellas sin ser el verdadero problema tienen el papel de distractoras, sesgan las investigaciones y generan una gama de conceptos de este fenómeno. Las macro variables del abandono o niveles de integración consiguen simplificar la visión y, a partir de ello alcanzar resultados que puedan trabajarse con más detenimiento.

El nivel de integración académico es el factor determinante del abandono, en la evidencia generada en este trabajo se confirma la relación negativa entre estas dos macro variables, por lo tanto el abandono de los estudiantes matriculados en un sistema de estudios a distancia es una consecuencia clara de los bajos niveles de integración académica de los estudiantes.

La relación puede resultar muy simple ya que agrupa la información reduciendo la causa a una sola, pero tiene la ventaja de centrar la discusión no en el abandono sino en las causas del bajo nivel de integración académico, rendimiento académico o resultados, por lo tanto, el reto que se plantea a todos los agentes del sistema son las medidas que se deben tomar, sin que se afecte la calidad, para que la media de resultados tienda o converja al mínimo aceptable de buen rendimiento, puesto que esto influirá directamente en la motivación del estudiante.

El comportamiento del abandono y la permanencia en el Ecuador en el sistema de estudios superiores a distancia prueban, en primera instancia, las características planteadas en la teoría del abandono y principalmente en la caracterización.

Las condiciones de la caracterización es necesario someterlas a otras realidades o resultados, de tal forma que se puedan consolidar como parte de la teoría general del abandono.

\section{NOTAS}

1. Se considera que un estudiante de una cohorte abandona cuando no registra matrícula en el periodo siguiente al de su ingreso.

2. Información existente en el Sistema Nacional de Información de la Educación Superior, SNIESE, datos actualizados del número de estudiantes nuevos y totales del año 2012. Quito o8 de mayo de 2013. 
3. Edad promedio de salida de los estudiantes del bachillerato.

4. Comparación del número de asignaturas aprobadas con el número de asignaturas totales matriculados. El indicador está entre cero y uno, cero para el caso de los estudiantes que reprueban en todos los componentes y uno para aquellos estudiantes que aprueban todos los componentes:

5. Las preferencias en el momento de escoger una carrera apuntan a que, la mayoría opta por los programas académicos que tradicionalmente han tenido mayor demanda: Abogacía, Administración de Empresas, Ingeniería en Contabilidad y Auditoría, y Psicología.

6. El punto mínimo de buen rendimiento que establece la Universidad Técnica Particular es $28 / 40$ puntos que equivale al $70 \%$ de la nota.

7. La Universidad Técnica Particular de Loja tienen una escala de calificación cuantitativa y cualitativa en función a ella se obtuvo estos datos.

\section{REFERENCIAS BIBLIOGRÁFICAS}

Álvarez Manrique, J. M. (1997). Etiología de un Sueño o El Abandono de la Universidad por parte de los estudiantes por factores no académicos. Santa Fe de Bogotá, D.C.: Universidad Autónoma de Colombia.

Bennett, R. (2003). Determinants of Undergraduate Student Drop Out Rates in a University Business Studies Department. Journal of Further and Higher Education, 27 (2).

Bethencour, J. T.; Cabrera Pérez, L.; Hernández Cabrera, J.; Álvarez Pérez, P.; González Afonso, M. (2008). Variables psicológicas y educativas en el abandono universitario. Revista Electrónica de Investigación Psicoeducativa, 6 (16), (603-622).

Cabrera, L.; Bethencourt, J.T.; Alvarez Pérez, P.; González Afonso, M. (2006). El Problema del Abandono de los Estudios Universitarios.The dropout problem in University Study. Relieve, (171-203).

Casquero Tomás, A.; Gómez, M. L. (2010). Determinantes del abandono escolar temprano en España: Un análisis por género. Revista de Educación, (191 223).
Corominas Rovira, E. (2001). La transición a los estudios universitarios. Abandono o cambio en el primer año de universidad. Revista de Investigación Educativa, 19 (1), (127-151).

Donoso, S.; Schiefelbein, E. (2007). Análisis de los modelos explicativos de retención de estudiantes en la Universidad: Una visión desde la desigualdad social. Estudios Pedagógicos XXXIII, (7-27).

García Aretio, L. (2001). La educación a distancia, de la teoría a la práctica. Madrid: Ariel Educación.

González Afonso, M.; Álvarez Pérez, P.; Cabrera Pérez, L.; Bethencour, J. T. (2007). El abandono de los estudiantes universitarios: factores determinantes y medidas preventivas. Revista Española de Pedagogía, (71-85).

Kuna, H.; García-Martínez, R.; Villatoro, F. (2009). Identificación de Causales de Abandono de Estudios Universitarios. Uso de Procesos de Explotación de Información. Revista Iberoamericana de Tecnología en Educación y Educación en Tecnología, 5, (39-44).

Moncada, L.; Rubio, M. J. (2011). Determinantes inmediatos del 
rendimiento académico en los nuevos estudiantes matriculados en el sistema de educación superior a distancia del Ecuador: caso Universidad Técnica Particular de Loja. RIED. Revista Iberoamericana de Educación a Distancia, 14 (2), (77-95).

Romero Fernández, L. M.; Rubio Gómez, M.J. (2007). Sistema de Créditos Académicos UTPL - ECTS: Hacia el Espacio Común de Educación Superior ALCUE.

Rubio Gómez, M. J. (2009). Orientaciones y Metodología para la Educación a Distancia. Loja: Editorial de la Universidad Técnica Particular de Loja.
Rubio Gómez, M. J. (2011). Nuevas orientaciones y metodología para la educación a distancia. Loja - Ecuador: Universidad Técnica Particular de Loja.

Rubio Gómez, M. J. (2013). Guía general de educación a distancia. Loja: Ediloja.

Tinto, V. (2004). Definición de la deserción, cuestión de perspectivas. Jossey-Bass Inc. Publishers.

Toharia, L.; Albert, C. (2000). El abandono o la persistencia en los estudios universitarios. Papeles de economía española, (192-212).

\section{PERFIL ACADÉMICO Y PROFESIONAL DEL AUTOR}

Luis Moncada Mora. Maestro en Economía Aplicada por la Universidad Nacional Autónoma de México. Máster en Evaluación, Gestión y Dirección de la Calidad Educativa de la Universidad de Sevilla. Actualmente Docente investigador de la Universidad Técnica Particular de Loja en la Titulación de Economía, la línea principal de estudio es Economía de la Educación.

E-mail: lifmoncada@utpl.edu.ec

DIRECCIÓN POSTAL DEL AUTOR

Modalidad a Distancia Universidad Técnica Particular de Loja.

San Cayetano Alto, $\mathrm{s} / \mathrm{n}$

Loja (Ecuador)

Fecha de recepción del artículo: 07/11/13

Fecha de aceptación del artículo: 13/05/14

\section{Como citar este artículo:}

Moncada Mora, L. F. (2014). La integración académica de los estudiantes universitarios como factor determinante del abandono de corto plazo. Un análisis en el sistema de educación superior a distancia del Ecuador. RIED. Revista Iberoamericana de Educación a Distancia, volumen 17, $\mathrm{n}^{0}$ 2, pp. 173-196. 